\title{
Vibration-based damage detection in an aircraft wing scaled model using principal component analysis and pattern recognition
}

\author{
I.Trendafilova ${ }^{1)}$, M.P Cartmell ${ }^{2)}$, W. Ostachowicz ${ }^{3)}$ \\ ${ }^{1)}$ Department of Mechanical Engineering, University of Strathclyde \\ 75 Montrose str., Glasgow G1 1XJ, Scotland, UK \\ 2) Department of Mechanical Engineering, James Watt Building, University of Glasgow, Glasgow, \\ G12 8QQ, Scotland, UK. \\ 3) Institute of Fluid-Flow Machinery of Polish Academy of Sciences, ul.Fiszera 14, 80-952, \\ Gdansk, Poland
}

\begin{abstract}
This study deals with vibration-based fault detection in structures and suggests a viable methodology based on principal component analysis (PCA) and a simple pattern recognition (PR) method. The frequency response functions (FRF's) of the healthy and the damaged structure are used as initial data. A PR procedure based on the nearest neighbour principle is applied to recognise between the categories of the damaged and the healthy wing data. A modified PCA method is suggested here, which not only reduces the dimensionality of the FRF's but in addition makes the PCA transformed data from the two categories more differentiable. It is applied to selected frequency bands of FRF's which permits the reduction of the PCA transformed FRF's to two new variables, which are used as damage features. In this study the methodology is developed and demonstrated using the vibration response of a scaled aircraft wing simulated by a finite element (FE) model. The suggested damage detection methodology is based purely on the analysis of the vibration response of the structure. This makes it quite generic and permits its potential development and application for measured vibration data from real aircraft wings as well as for other real and complex structures.
\end{abstract}

Keywords: aircraft wing, damage detection, vibration-based health monitoring, principal component analysis, pattern recognition methods.

\section{Introduction and background.}


Vibration-based health monitoring (VHM) methods are global non-destructive testing methods, which are based on the fact that any changes introduced in a structure (including damage) change its physical properties, and this in turn changes the structural vibration response. For most applications VHM is applied using the modal characteristics of the structure and especially its lower resonant frequencies, which are easy to measure from experiment [1-4]. Unfortunately in a lot of cases as has been reported in several studies these frequencies turn out to be insensitive to damage, and especially to lower levels of damage $[1,2,5,6]$. The mode shapes present a further alternative for damage assessment, but they are difficult to measure and their estimate from data may involve substantial inaccuracies $[1,3,4,6]$. Another option are the updating methods, which rely on a certain model of the structure, which is in most cases linear and thus might not be accurate enough especially for a more complicated structure and/or when nonlinear effects are present

\section{$[1,4,6]$.}

Intrinsically the problems for vibration-based damage detection, quantification and localization are pattern recognition problems since they suggest the discrimination between two or more signal categories, i.e. signals coming from an intact structure and those from a damaged structure, or signals coming from a structure with different damage levels or locations $[7,8,9]$. This perspective is used in the present study, and it presents another possible approach for damage diagnosis, which is a rather generic one since it is based solely on the structural vibration response and thus does not use any assumptions of model or linearity. This type of purely data-based VHM methods are relatively new but the existing research reports promising results and demonstrates their potential $[7,8,10-13]$. Such an approach will include a signal processing stage to extract damage sensitive features and thereby to prepare the necessary environment for the next recognition (categorisation) stage. The feature extraction stage aims at the reduction of the originally measured data but also at forming suitable features to be used for the recognition stage. Different feature extraction methods have been suggested for damage detection purposes [1,7-9]. PCA is a powerful data reduction method and its application for structural vibration and VHM is suggested in several papers [10-12]. This study suggests the use of a modified PCA method, which does not only reduce the dimension of the measured FRF's, it also makes the transformed data from different categories more recognisable. This modified PCA approach has been suggested for categorical data and it can be proven that it reduces the intercategory distance while in the same time increasing the between-category distance of the PCA transformed data $[14,15]$. It should be noted also that in this particular case PCA is applied to two frequency bands of 
FRF's which reduces the dimension of the original data and eventually allows the use of only two PCA transformed variables. This enables the visualisation of the PCA projected data in a plane and facilitates significantly the next recognition stage. The actual recognition stage between the data from damaged and non-damaged cases in the suggested VHM method is performed by a simple PR method based on the nearest neighbour principle. Several papers suggest the use of neural network-based methods for this recognition stage $[12,13,14]$. In some of these papers PCA transformed FRF's are used to train the networks $[12,13]$. We would like to stress on the simplicity of the recognition method used here, which is much easier and requires much less computer resources compared to a neural network. Thus the main objective of this paper is to present a feasible method for structural VHM, which 1) reduces the dimension of the initial FRF data and transforms it into new damage features and 2) employs a simple PR method for the actual damage detection (recognition) stage.

Another motivation for this paper and especially the development of the method for aircraft wings is the potential that VHM methods may hold for monitoring aircraft structures and elements. Aircraft accidents due to unnoticed faults, especially when they happen in-flight, can have disastrous and fatal consequences and it is a major concern of the aircraft companies to prevent unacceptable structural failures from accidental, environmental and fatigue damage. VHM is not currently incorporated in schemes for monitoring aircraft structures, although it is expected to play a significant role since such methods can bring major benefits like increased safety, extended structural life, reduced inspections, reduced aircraft weight, decreased operating costs, detection of cracks, corrosion and fatigue damage in areas with restricted access $[5,16]$. This paper is an early stage of an investigation, based on FE simulated results, oriented towards the development of a practical global monitoring method for real aircraft structures. As a next step in this development the suggested VHM method is being currently applied to measured data from an experimental model of a scaled aircraft wing.

\section{The damage detection method}

As it was mentioned above this method uses principal component analysis in the frequency domain as a feature selection procedure.

PCA is applied when one has a number $n$ of realisations of a multidimensional (p-dimensional) variable. In this particular case the FRF's for $p$ different frequencies are packed to form a vector $\mathbf{h}$, 
$\mathbf{h}=\left[\mathbf{h}_{\mathbf{1}}, \mathbf{h}_{\mathbf{2}}, \ldots, \mathbf{h}_{\mathbf{p}}\right]^{T}$. Then $n$ realisations of the vector $\mathbf{h}$, can be obtained from different measurement points and/or measurements. Accordingly we have $n$ realisations of the p-dimensional vector variable $\mathbf{h}$, which come from two different categories corresponding to 'no-damage' and 'damage' states. The idea of PCA is to transform the original multidimensional data to new vector variables $\mathbf{c}$ with a smaller dimension $k$, $k<<p$. This is done using the autocorrelation matrix of the $n$ realisations of the vector $\mathbf{h}$. In the case of categorical data like this one it is better to use the generalised autocorrelation matrix rather than the autocorrelation matrix $[10,14,15]$. It is calculated following the relation:

$$
\mathbf{R}=\sum_{i=1}^{M} p\left(\omega_{i}\right) E\left(\overline{\mathbf{h}}_{\mathbf{i}} \overline{\mathbf{h}}_{\mathbf{i}}^{T}\right)
$$

where $M$ is the number of categories (in this case $\mathrm{M}=2$ ), $p\left(\omega_{\imath}\right)$ is the a priori probability for the category $\omega_{l}$, the superscript ' $T$ ' stands for transpose, $E$ denotes the mathematical expectation, and $\overline{\mathbf{h}}_{\mathbf{i}}$ is a standardised vector from the $i$-th category. Then the eigenvalues of the generalised correlation matrix $\mathbf{R}$, and their corresponding eigenvectors, are found. The first $k$ largest eigenvalues and their associated eigenvectors $\Phi_{\mathrm{i}}$ are taken. The vectors $\Phi_{\mathrm{i}}$ are packed to form the transformation matrix $\Phi$

$$
\mathbf{\Phi}=\left[\Phi_{1}, \Phi_{2}, \ldots, \Phi_{k}\right]
$$

A vector $\mathbf{h}$ is transformed into a new feature $\mathbf{c}$ vector via

$$
\mathbf{c}=\boldsymbol{\Phi}^{\prime} \mathbf{h},
$$

where $\boldsymbol{\Phi}^{\prime}$ is the transpose of $\boldsymbol{\Phi}$.

The primary idea of PCA is to transform the original vector $\mathbf{h}$ into a new vector $\mathbf{c}$, which has a smaller dimension $k, k<<p$. The choice of the $k$ new variables is based on the variance that they are responsible for. When applied to categorical data the modified PCA used here, which employs the generalised autocorrelation matrix, is expected to have other advantageous properties since it decreases the inter-class variance at the same time as increasing the between-class variance. This results in 'clustering' the new PCA transformed vectors or bringing together the vectors from the same category while in the same time increasing the distance between vectors from different categories $[11,15,16]$. In this particular case the procedure is aimed to cluster, or group, the vectors from the undamaged and the damaged categories and to 
separate the two groups. The obtained transformed vector-variables c are further used as features for recognising between the data from damaged and undamaged cases.

One rather intuitive approach is to classify each vector $\mathbf{c}$ into the category to which its "nearest neighbour (NN)", the vector nearest to c, belongs $[10,13]$. This is the so-called $1-N N$ classifier. In this study a nearest neighbour is found on the basis of Euclidean distance. The Euclidean distance between two feature vectors c and $\mathbf{c}_{\mathbf{i}}$ is defined as:

$$
D_{i}=\left\|\mathbf{c}-\mathbf{c}_{\mathbf{i}}\right\|=\sqrt{\left(\mathbf{c}-\mathbf{c}_{\mathbf{i}}\right)^{T}\left(\mathbf{c}-\mathbf{c}_{\mathbf{i}}\right)}
$$

The 1-NN classifier finds the nearest neighbor of $\mathbf{c}, \mathbf{c}_{\mathbf{i}}$, and assigns $\mathbf{c}$ to the class of $\mathbf{c}_{\mathbf{i}}$. So in order to apply this classifier one needs a 'training' sample of vectors $\mathbf{c}_{\mathbf{j}}$, among which the classifier will look for the NN. The construction of the training sample is discussed later. In the case of a large number of training data the 1$N N$ rule gives a rather low probability for error which is close to the Bayesian [13]. This study uses the 1-NN classifier to classify the new feature vectors $\mathbf{c}$ as damaged or non-damaged.

\section{The FE model and the representation of damage.}

At this stage a rather simple finite element model of a scaled aircraft wing is used to predict its vibration response ( Fig.1). The model is made of 200 aluminium alloy solid elements with three degrees of freedom. The number of elements is established after a convergence analysis. The purpose of the model is to predict from general principles, the modal characteristics and the vibration response of the structure. The wing model was divided into five damage volumes, V1-V5, as presented in Figure 1.

Two types of damage are considered- in the form of cracks and distributed damage. Cracks are introduced by disbanding a number of elements. The crack size is changed by varying the number of disbanded elements. These are supposed to represent very narrow, one dimensional, cracks, for which only the length can be varied.

It is known that most damage scenarios such as corrosion, erosion and distributed cracks in composites, generally cause stiffness reduction. Accordingly, in this study distributed damage is introduced by reducing the stiffness of a whole volume of the wing. The extent of the distributed damage is controlled by introducing different stiffness reductions. 
Damage was introduced in all the five damage volumes (V1-V5) consecutively. It was found that the vibration response in the first volume V1, which is close to the root of the wing, was the one most affected by damage (see Fig.1). The current investigation aims to develop and validate the suggested method for FE simulated data from an aircraft wing and at this stage only damage in V1 is considered.

\section{Damage detection in the aircraft wing model}

The FRF's for 50 equidistantly distributed points covering the wing surface were found for excitation applied at the midpoint of the wing model (Fig.1). It was observed that the values of the FRFs close to the peaks are most affected by damage. The two frequency bands, which undergo the maximum changes are those around the peaks of $35 \mathrm{~Hz}$ and $112 \mathrm{~Hz}$. So only 43 frequency lines, between $25 \mathrm{~Hz}$ and $45 \mathrm{~Hz}$ and between $101 \mathrm{~Hz}$ and $122 \mathrm{~Hz}$, were used to make compressed FRF vectors. Then a number of realizations of the FRF vectors are obtained by taking the FRF's corresponding to the 50 measurement points and by adding Gaussian noise with RMS levels between $1 \%$ and 3\%. The modified PCA decomposition (eq.(1) to (3)) was first applied to the whole frequency range (the vectors made of 1000 frequency lines of the FRF's) and it was concluded that in this case the first two principal components (PC's) accounted for about 70\% of the whole variance while the first 5 PC's contained $93.20 \%$ of the whole variance. Then the PCA transform was applied to the compressed FRF vectors made of the 43 frequency lines as explained above. In this case the first two principal components were responsible for $92.11 \%$ of the whole variance. For this reason it was decided to use the compressed frequency vectors $\mathbf{h}$ made of 43 frequency lines and retain the first two principal components partly for visualization purposes but also because they accounted for a considerable part of the whole variance $(92.11 \%)$. The whole damage detection procedure works according to the following algorithm:

1. A "training" sample is generated from data obtained for both damage states. (The word "training" is used here and further in the text to indicate that FRF's with known categories, i.e. calculated for known, damaged or non damaged, states, are used.) To generate it $n$ realizations of FRF's corresponding to non-damaged state and to different damage levels are obtained as was explained above (using the FRF's from the 50 points on the wing surface and by adding Gaussian noise with

RMS levels between 1-3\%). Then only the 43 frequency lines considered above are used to obtain the training sample of $n$ compressed (43-dimensional) FRF vectors $\mathbf{h}$. 
2. The obtained training sample of $n$ vectors $\mathbf{h}$ is used to calculate the autocorrelation matrix $\mathbf{R}$ (equation (1)) and the transformation matrix $\boldsymbol{\Phi}$ corresponding to the first two eigenvalues (eq. (2)).

3. The training vectors $\mathbf{h}$ are then PCA transformed into new feature vectors $\mathbf{c}$ according to (3). The obtained feature vectors $\mathbf{c}$ form two sets of vectors corresponding to the two categories of data and to the states of damage and no-damage.

4. When new FRF data is presented for recognition, it is first compressed by taking only the above discussed 43 frequency lines and the obtained vector $\mathbf{h}^{*}$ is PCA transformed into a feature vector $\mathbf{c}^{*}$. Then the recognition procedure is carried out and $\mathbf{c} *$ is classified to the class of its nearest neighbour according to the $1-N N$ rule.

\section{Some results}

Cracks are introduced as explained above and the length of the crack varies between $0.01 w$ to $0.1 w$, where $w$ is the wing width, which is from $5 \mathrm{~mm}$ to $50 \mathrm{~mm}$ in the example taken here.

In a previous study [5] we investigated the sensitivity of the natural frequencies of a similar FE model of an aircraft wing to damage and the results for this FE model confirm that the first ten natural frequencies show very small changes to both types of damage considered. The changes are less than $0.3 \%$ for a $50 \mathrm{~mm}$ crack and they are still less than $1 \%$ for a crack of length equal to half of the wing width, which is $250 \mathrm{~mm}$. Our results for stiffness reduction show that $50 \%$ stiffness reduction in V1 causes changes of up to about $1 \%$. It can be therefore concluded on the basis of the FE results that the first ten natural frequencies are not good candidates for damage features for this aircraft wing model.

At this point the method suggested in the previous section is applied following the algorithm detailed above. A training sample of $1100 \mathbf{h}$ vectors is generated according to step 1 in the above algorithm: 100 of these vectors correspond to undamaged state and the rest - to damaged state with different crack lengths (Table 1). The length of the cracks varies from $5 \mathrm{~mm}$ to $50 \mathrm{~mm}$ and 100 training vectors are generated for each crack length. Steps 2 and 3 from the above algorithm are performed to obtain the PCA transformed feature vectors. Figure 2 presents the distribution of the new feature vectors from both categories. The two categories in Figure 2 are clearly distinguishable. 
Another sample of $1100 \mathrm{FRF}$ vectors is then prepared the same way as the training sample to be used for testing purposes. The testing sample is used to test the categories/states of the FRF's in it and compare them to their true categories. The new feature vectors are obtained and classified as damaged or undamaged according to step 4 of the above algorithm. Table 1 shows the number of correctly recognized states for the different crack sizes.

Distributed damage in V1 is introduced as explained above and nine levels of damage representing from $10 \%$ to $50 \%$ stiffness reduction are considered (Table 2). A training sample of $1000 \mathbf{h}$ vectors is made for distributed damage according to step 1 of the above algorithm, in a similar way to the one used for crack detection. This comprises 100 vectors from the undamaged state and 100 from each of the nine damage levels from $10 \%$ to $50 \%$ stiffness reduction. Figure 3 shows the distribution of the new feature vectors from the training sample corresponding to the damaged and the undamaged states (obtained following steps 2 and 3 of the above algorithm). Again the clustering effect is evident.

Another 1000 realizations of the FRF vectors are created to form the testing sample for distributed damage. It is formed in the same way as the training sample discussed above. Table 2 shows the number of correctly recognized states (damage or undamaged) for the different damage levels (including the undamaged level).

\section{Conclusions}

(1) This paper suggests a viable method for structural damage detection based on a pattern recognition perspective, which recognizes between PCA transformed FRF's from a structure in damaged and in non-damaged state. It uses a modified PCA transform to obtain low-dimensional damage features which help the recognition between the two categories.

(2) The suggested methodology is a generic one and permits potential development for measured FRF's as well as for other structures

(3) The use of selected frequency bands of FRF's makes the methodology structure dependent and requires its development for each structure which will depends on and include the selection of damage sensitive FRF frequency bands. 
(4) In the present study the methodology is developed for a scaled model of an aircraft wing. The results are based on a FE simulation of the FRF's of the model. Its further development as a practical monitoring method requires testing and validation for measured FRF data.

(5) The results indicate that the combination of the modified PCA transform and the pattern recognition procedure suggested provide a suitable methodology for structural damage detection.

(6) From the provided numerical experiments it can be concluded that the approach suggested here worked quite well for the used FE model of an aircraft wing. It proved capable of detecting both types of damage in the wing model at quite a low level. It is obvious that the method can be applied with a very high success rate to simulated FEA results, to which noise of between 1 and $3 \%$ has been added. These results should be confirmed from experimental measurements, as well, before the method can be deemed suitable for practical applications.

Acknowledgments: The authors of this work would like to gratefully acknowledge the financial support of NATO grant CBP.EAP.CLG.981517.

\section{References}

[1] S.W.Doebling, C.R. Farrar and M.B. Prime, A Summary Review of Vibration-Based Damage Identification Methods, The Shock and Vibration Digest, Vol. 30, No. 2, (1998), pp. 91-105.

[2] B.Lazarov and I.Trendafilova, An investigation on Vibration-based damage diagnosis in thin plates, Structural Health Monitoring 2004, Proc. of the II European Workshop, Munich (July 2004) pp 76-82.

[3] P. Verboven, E. Parloo, P. Guillaume and M. Van Overmeire: Autonomous structural health monitoring-part I: Modal parameter estimation and tracking, Mechanical Systems and Signal Processing, vol. 16(4), (2002), pp 637-657.

[4] N.Hu, X Wang, H. Fukunaga, Z.H Yao, H.X. Zhang and Z.S. Wu, Damage assessment of structures using modal test data, International Journal of Solids and Structures, vol.38/18 (May 2001), pp.3111-3126. 
[5] I. Trendafilova, A study on vibration-based damage detection and location in an aircraft wing scaled model, Applied Mechanics and Materials, vol 3-4 (2005), pp 309-314

[6] M.J. Sundaresan A. Ghoshal J. Li M.J. Schulz P.F. Pai and J.H Chung, Experimental damage detection on a wing panel using vibration deflection shapes, Structural Health Monitoring, 2(3), (2003) pp 243-256.

[7] H. Sohn, Charles R. Farrar, Norman F. Hunter, and Keith Worden, Structural Health Monitoring Using Statistical Pattern Recognition Techniques, ASME Journal of Dynamic Systems, Measurement and Control: Special Issue on Identification of Mechanical Systems, , vol.123, issue 4 (2001), pp 706712.

[8] G. Manson K.Worden and D. Allman, Experimental validation of a structural health monitoring methodology: part II. Novelty detection on a gnat aircraft, Journal of Sound and Vibration, 259(2), (2003) pp 345-363.

[9] I.Trendafilova and W.Heylen, Categorization and Pattern Recognition methods for Damage Localization from Vibration Measurements, Mechanical Systems and Signal Processing, vol 17 (4) (2003), p. 825-836.

[10] I.Trendafilova, M. Imbabi, Novel Health Monitoring Procedure for Reinforced Concrete Slabs, AMM Applied Mechanics and Materials, vol 1 (2004) p. 65-71.

[11] Y.Q. Ni_, X.T. Zhou, J.M. Ko, Experimental investigation of seismic damage identification using PCA-compressed frequency response functions and neural networks, Journal of Sound and Vibration, 290 (2006) p. 242-263.

[12] C. Zang and M. Imregun, Structural damage detection using artificial neural networks and measured FRF data reduced via principal component projection, Journal of Sound and Vibration242(5), (2001), p. $813-827$

[13] T. Marwala and H. E. M. Hunt, Fault identification using finite element models and neural networks, Mechanical Systems and Signal Processing 13, 1999 p. 475-490.

[14] I.T.Jolliffe, Principal component analysis Springer-Verlag, New York,(2002).

[15] J.T. Tou, R. C. Gonzales, Pattern recognition principles Addison-Wesley Publ. Comp (1974) 
[16] H.-J. Schmidt, J. Telgkamp and B. Schmidt-Brandecker, Application of structural health monitoring to improve efficiency of aircraft structure, Structural Health Monitoring 2004, Proc. of the II European Workshop, Munich (July 2004) p.11.

\section{List of Figure captions}

Figure 1. Schematic of the wing model

Figure 2. Distribution of the new feature vectors for undamaged and for cracked wing

Figure 3. Distribution of the new feature vectors for distributed damage in V1

\section{List of Table captions}

Table 1. Number of correctly recognised cases (out of 100) for different crack sizes.

Table 2. Number of correctly recognised cases (out of 100) for different levels of distributed damage. 


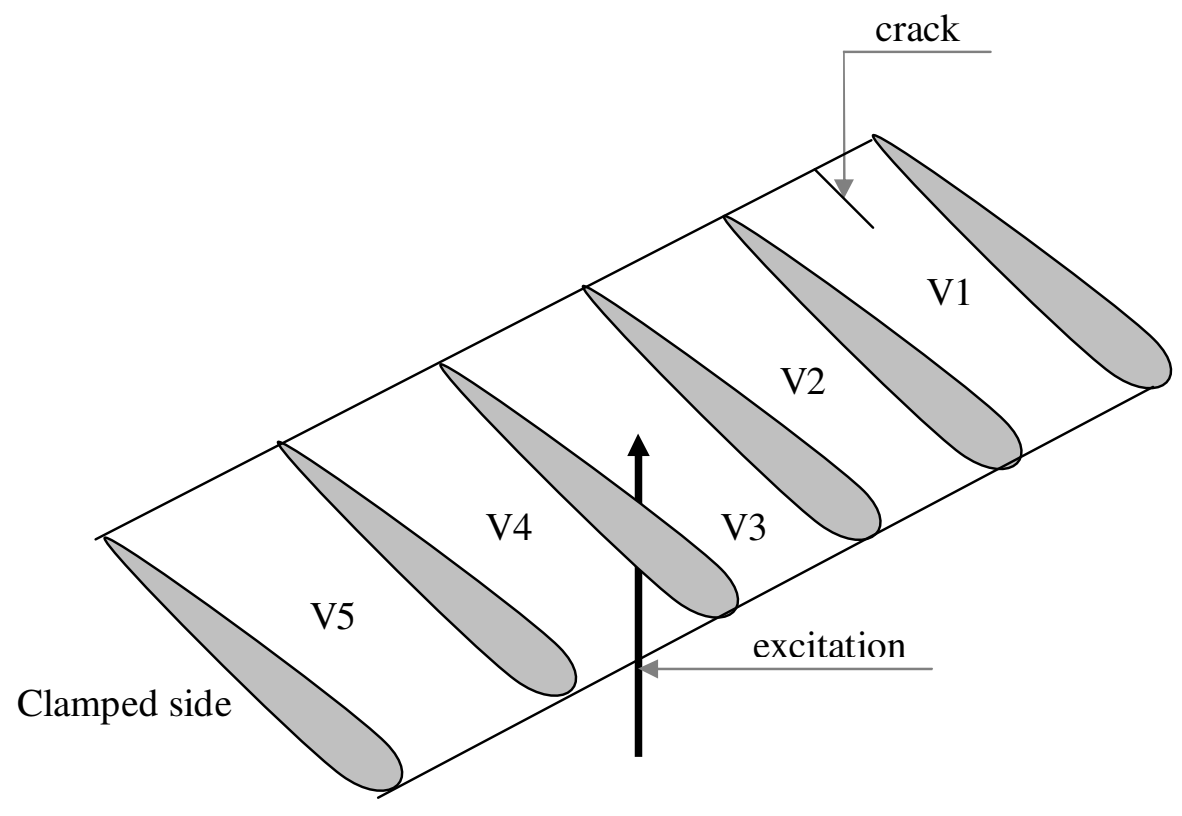

Figure 1. Schematic of the wing model 


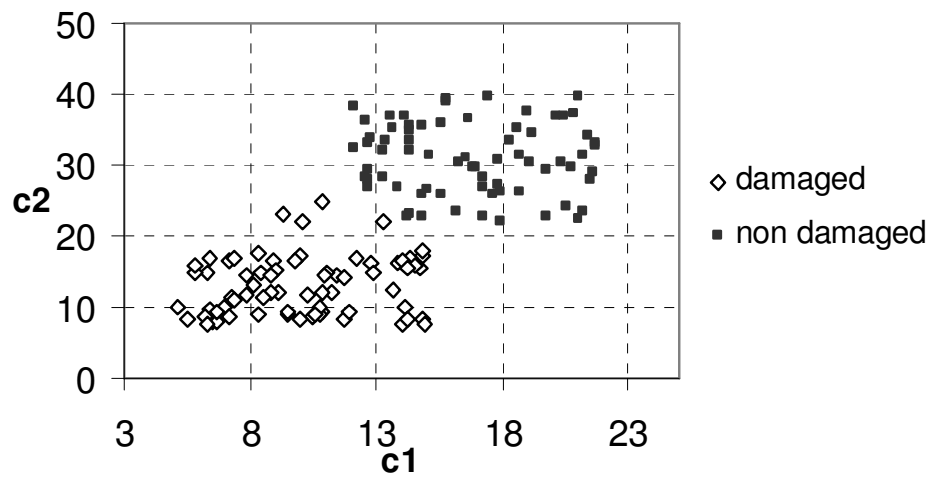

Figure 2. Distribution of the new feature vectors for undamaged and for cracked wing 


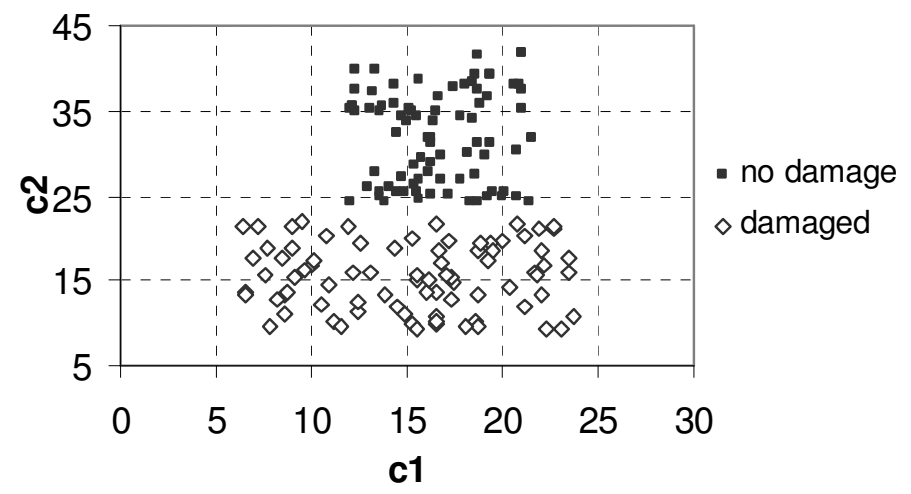

Figure 3. Distribution of the new feature vectors for distributed damage in V1 


\begin{tabular}{|l|l|l|l|l|l|l|l|l|l|l|l|}
\hline & \multicolumn{10}{|c|}{ Crack in mm } \\
\hline & 0 & 5 & 10 & 15 & 20 & 25 & 30 & 35 & 40 & 45 & 50 \\
\hline Number & & & & & & & & & & & \\
correctly & 99 & 90 & 94 & 96 & 97 & 95 & 98 & 99 & 100 & 99 & 100 \\
recognized & & & & & & & & & & & \\
\hline
\end{tabular}

Table 1. Number of correctly recognised cases (out of 100) for different crack sizes. 


\begin{tabular}{|l|l|l|l|l|l|l|l|l|l|l|}
\hline & \multicolumn{10}{|c|}{ Stiffness reduction in \% } \\
\hline & 0 & 10 & 15 & 20 & 25 & 30 & 35 & 40 & 45 & 50 \\
\hline $\begin{array}{l}\text { Number } \\
\text { correctly } \\
\text { recognized }\end{array}$ & 99 & 94 & 98 & 99 & 98 & 98 & 100 & 99 & 99 & 100 \\
\hline
\end{tabular}

Table 2. Number of correctly recognised cases (out of 100) for different levels of distributed damage. 